VoL. 75 (2007) [313-320]

\title{
FINITE GROUPS IN WHICH SOME PROPERTY OF TWO-GENERATOR SUBGROUPS IS TRANSITIVE
}

\author{
Costantino Delizia, Primož Moravec and Chiara Nicotera
}

\begin{abstract}
Finite groups in which a given property of two-generator subgroups is a transitive relation are investigated. We obtain a description of such groups and prove in particular that every finite soluble-transitive group is soluble. A classification of finite nilpotent-transitive groups is also obtained.
\end{abstract}

\section{INTRODUCTION}

Let $\mathfrak{X}$ be a group theoretical class. A group $G$ is said to be $\mathfrak{X}$-transitive (or an $\mathfrak{X}$ group) if for all $x, y, z \in G \backslash\{1\}$ the relations $\langle x, y\rangle \in \mathfrak{X}$ and $\langle y, z\rangle \in \mathfrak{X}$ imply $\langle x, z\rangle \in \mathfrak{X}$. In graph theoretical terms, let $\Gamma_{\mathfrak{X}}(G)$ be the simple graph whose vertices are the nontrivial elements of $G$, and $a$ and $b$ are connected by an edge if and only if $\langle a, b\rangle \in \mathfrak{X}$. Then $G$ is an $\mathfrak{X T}$-group precisely when all the connected components of $\Gamma_{\mathfrak{X}}(G)$ are complete graphs. Several authors have studied $\mathfrak{X T}$-groups for some special classes $\mathfrak{X}$. When $\mathfrak{X}$ is the class of all Abelian groups, these groups are also known as commutative-transitive groups or CT-groups. Weisner [10] has shown that finite $C \mathrm{~T}$-groups are either soluble or simple. Finite nonabelian simple $C$ T-groups have been classified by Suzuki [6]. These are precisely $\operatorname{PSL}\left(2,2^{f}\right)$, where $f>1$. A characterisation of finite soluble $C T$-groups has been given by $\mathrm{Wu}[11]$ who has also obtained information on locally finite $C \mathrm{~T}$-groups and polycyclic $C$ T-groups. When $\mathfrak{X}=\mathfrak{N}_{c}$, the class of all groups which are nilpotent of class $\leqslant c$, similar results have been obtained in $[\mathbf{1}]$.

The purpose of this note is to obtain a description of finite $\mathfrak{X T}$-groups for the group theoretical classes $\mathfrak{X}$ having the following properties:

( $\star$ I $\mathfrak{X}$ is subgroup closed, it contains all finite Abelian groups and is bigenetic in the class of all finite groups.

Here a class $\mathfrak{X}$ is said to be bigenetic (a terminology due to Lennox [4]) in the class of all finite groups when a finite group $G$ is in $\mathfrak{X}$ if and only if all its two-generator subgroups are. Examples of classes satisfying $(\star)$ are the class of all Abelian groups, all nilpotent

\section{Received 30th October, 2006}

The second author was partially supported by G.N.S.A.G.A. He also wishes to thank the Department of Mathematics and Informatics at the University of Salerno for its excellent hospitality.

Copyright Clearance Centre, Inc. Serial-fee code: 0004-9727/07 \$A2.00+0.00. 
groups, all supersoluble groups and all soluble groups. First we show that if $\mathfrak{X}$ is a class satisfying $(\star)$, then every finite $\mathfrak{X T}$-group which does not belong to $\mathfrak{X}$ is either a Frobenius group with kernel and complement belonging to $\mathfrak{X}$, or it has no normal $\mathfrak{X}$-subgroups, that is, it is $\mathfrak{X}$-semisimple as defined in [5]. We also show that in several cases, for example, in the soluble or supersoluble case, the second possibility does not occur. As a consequence we obtain that a finite group is soluble if and only if it is soluble-transitive. In the case when $\mathfrak{X}=\mathfrak{N}$, the class of all nilpotent groups, there exist simple $\mathfrak{N T}$-groups. We obtain a complete classification of finite $\mathfrak{N T}$-groups which generalises some results of [11].

\section{Results}

Given a group theoretical class $\mathfrak{X}$, let $R_{\mathfrak{X}}(G)$ be the product of all normal $\mathfrak{X}$ subgroups of $G$ (the $\mathfrak{X}$-radical of $G$ ). In general $R_{\mathfrak{X}}(G)$ does not belong to $\mathfrak{X}$. Our first result shows that this is however true within the class of all finite $\mathfrak{X}$-transitive groups when $\mathfrak{X}$ satisfies the properties $(\star)$.

LEMMA 2.1 . Let $\mathfrak{X}$ be a class of groups satisfying $(\star)$ and let $G$ be a finite $\mathfrak{X T}$ group. Then $R_{\mathfrak{X}}(G)$ is an $\mathfrak{X}$-group.

Proof: Let $M$ and $N$ be normal $\mathfrak{X}$-subgroups of $G$. It suffices to show that $M N$ also belongs to $\mathfrak{X}$. Suppose first that $M \cap N \neq 1$ and let $x \in M \cap N \backslash\{1\}$. First note that for any $m \in M \backslash\{1\}$ and $n \in N \backslash\{1\}$ we have that $\langle m, x\rangle$ and $\langle x, n\rangle$ belong to $\mathfrak{X}$. As $G$ is an $\mathfrak{X T}$-group, we conclude that $\langle m, n\rangle$ is an $\mathfrak{X}$-group. Now let $m_{1}, m_{2} \in M \backslash\{1\}$ and $n \in N \backslash\{1\}$. We may suppose that $m_{1} n \neq 1$. Then $\left\langle m_{1} n, m_{1}\right\rangle=\left\langle m_{1}, n\right\rangle$ is in $\mathfrak{X}$ and $\left\langle m_{1}, m_{2}\right\rangle$ is in $\mathfrak{X}$. Thus it follows that $\left\langle m_{1} n, m_{2}\right\rangle$ also belongs to $\mathfrak{X}$. Similarly we can prove that $\left\langle m n_{1}, n_{2}\right\rangle$ is in $\mathfrak{X}$ for every $m \in M \backslash\{1\}$ and $n_{1}, n_{2} \in N \backslash\{1\}$. Now take $m_{1}, m_{2} \in M \backslash\{1\}$ and $n_{1}, n_{2} \in N \backslash\{1\}$ and suppose that $m_{1} n_{1} \neq 1, m_{2} n_{2} \neq 1$. Then $\left\langle m_{1} n_{1}, m_{2}\right\rangle \in \mathfrak{X},\left\langle m_{2}, m_{2} n_{2}\right\rangle \in \mathfrak{X}$, hence $\left\langle m_{1} n_{1}, m_{2} n_{2}\right\rangle$ belongs to $\mathfrak{X}$. This shows that every two-generator subgroup of $M N$ belongs to $\mathfrak{X}$. Since $\mathfrak{X}$ is bigenetic in the class of all finite groups, we get that $M N$ is an $\mathfrak{X}$-group, as required.

Suppose now that $M \cap N=1$. Then $[M, N]=1$. As above it suffices to prove that every two-generator subgroup of $M N$ is in $\mathfrak{X}$. At first let $m_{1}, m_{2} \in M \backslash\{1\}$ and $n \in N \backslash\{1\}$. Then the groups $\left\langle m_{1} n, n\right\rangle=\left\langle m_{1}, n\right\rangle$ and $\left\langle n, m_{2}\right\rangle$ are Abelian, hence they belong to $\mathfrak{X}$. By the transitivity we have that $\left\langle m_{1} n, m_{2}\right\rangle$ belongs to $\mathfrak{X}$. Similar argument shows that $\left\langle m n_{1}, n_{2}\right\rangle \in \mathfrak{X}$ for every $m \in M \backslash\{1\}$ and $n_{1}, n_{2} \in N \backslash\{1\}$. From this it follows that if $m_{1}, m_{2} \in M \backslash\{1\}$ and $n_{1}, n_{2} \in N \backslash\{1\}$, then $\left\langle m_{1} n_{1}, m_{2}\right\rangle$ and $\left\langle m_{2}, m_{2} n_{2}\right\rangle$ are in $\mathfrak{X}$, hence $\left\langle m_{1} n_{1}, m_{2} n_{2}\right\rangle$ is also in $\mathfrak{X}$. This concludes the proof.

TheOREM 2.2. Let $\mathfrak{X}$ be a class of groups satisfying ( $*$ ). Let $G$ be a finite XT-group. Then one of the following holds.

(i) $G$ belongs to $\mathfrak{X}$.

(ii) $G$ is $\mathfrak{X}$-semisimple. 
(iii) $G$ is a Frobenius group with kernel and complement both belonging to $\mathfrak{X}$.

Proof: Let $R$ be the $\mathfrak{X}$-radical of $G$. By Lemma $2.1, R$ belongs to $\mathfrak{X}$. If $R=G$, then $G$ belongs to $\mathfrak{X}$. If $R=1$, then $G$ is $\mathfrak{X}$-semisimple. So from now on we assume that $1 \neq R \neq G$.

Let $y \in R \backslash\{1\}$ and suppose that there exists $a \in C_{G}(y) \backslash R$. Then $\langle a, y\rangle$ is Abelian, hence it belongs to $\mathfrak{X}$. As $R$ is an $\mathfrak{X}$-group and $G$ is an $\mathfrak{X}$-group, we have that $\langle a, h\rangle$ is in $\mathfrak{X}$ for every $h \in R$. By conjugation we get that $\left\langle a^{x}, h\right\rangle \in \mathfrak{X}$ for every $x \in G$ and $h \in R$. Since $G$ is an $\mathfrak{X}$-group, we get that

$$
\left\langle a^{x}, a^{z}\right\rangle \in \mathfrak{X}
$$

for every $x, z \in G$. We claim that $\langle u, v\rangle \in \mathfrak{X}$ for every $u, v \in a^{G}$. To prove this, we first introduce some notation. For $u \in a^{G}$ let $r$ be the smallest integer such that $u$ can be written as $a^{ \pm g_{1}} \cdots a^{ \pm g_{r}}$ for some $g_{1}, \ldots, g_{r} \in G$. Then we say that $u$ is of weight $r$ and denote $\operatorname{wt}(u)=r$. The proof of our claim goes by induction on $\operatorname{wt}(u)+\operatorname{wt}(v)$. If $\mathrm{wt}(u)+\mathrm{wt}(v) \leqslant 2$, then the claim follows from (1). Suppose that the claim holds true for all $u, v \in a^{G}$ with $\operatorname{wt}(u)+\operatorname{wt}(v) \leqslant l$. Let now $u, v \in a^{G}$ be such that $\operatorname{wt}(u)+\operatorname{wt}(v)=l+1$. Without loss of generality we may assume that $\operatorname{wt}(u)>1$ and $v \neq 1$. Then we can write $u=u^{\prime} a^{ \pm g}$ for some $g \in G$ and $u^{\prime} \in a^{G} \backslash\{1\}$ with $\operatorname{wt}\left(u^{\prime}\right)=\operatorname{wt}(u)-1$. We have that $\left\langle u, a^{g}\right\rangle=\left\langle u^{\prime}, a^{g}\right\rangle$ belongs to $\mathfrak{X}$ by the induction assumption. For the same reason we have that $\left\langle a^{g}, v\right\rangle \in \mathfrak{X}$. As $G$ is an $\mathfrak{X T}$-group, we conclude that $\langle u, v\rangle$ belongs to $\mathfrak{X}$. This proves that every two-generator subgroup of $a^{G}$ belongs to $\mathfrak{X}$. As $\mathfrak{X}$ is bigenetic in the class of all finite groups, we get $a^{G} \in \mathfrak{X}$, hence $a \in R$, a contradiction. By Satz 8.5 in [2] we have that $G$ is a Frobenius group and $R$ is its kernel. In particular, it follows from here that $R$ is nilpotent. Let $H$ be its complement. Then $H$ is an $\mathfrak{X T}$-group with nontrivial centre. It follows from here that every two-generator subgroup of $H$ belongs to $\mathfrak{X}$, hence $H \in \mathfrak{X}$.

A characterisation of $\mathfrak{X}$-semisimple $\mathfrak{X} T$-groups is usually not easy and depends heavily on a choice of the class $\mathfrak{X}$; see $[1,6,11]$. In the case of Frobenius groups we provide a general characterisation of $\mathfrak{X T}$-groups. At first we prove the following technical result.

LEммA 2.3. Let $\mathfrak{X}$ satisfy $(\star)$. Let $G$ be a finite $\mathfrak{X T}$-group and $H$ an $\mathfrak{X}$-subgroup of $G$. Then

$$
C_{G}^{\mathfrak{x}}(H)=\{x \in G:\langle x, h\rangle \in \mathfrak{X} \text { for some } h \in H \backslash\{1\}\}
$$

is an $\mathfrak{X}$-subgroup of $G$ containing $H$.

Proof: Clearly $C_{G}^{x}(H)$ contains $H$. Let $x, y \in C_{G}^{x}(H) \backslash\{1\}$. Then there exist $h, k \in H \backslash\{1\}$ such that $\langle x, h\rangle \in \mathfrak{X}$ and $\langle y, k\rangle \in \mathfrak{X}$. Since $\langle h, k\rangle \in \mathfrak{X}$, we get that $\langle x, y\rangle$ also belongs to $\mathfrak{X}$. If $x y \neq 1$, then $\langle x y, y\rangle=\langle x, y\rangle$ belongs to $\mathfrak{X}$, hence also $\langle x y, k\rangle \in \mathfrak{X}$. Thus $x y \in C_{G}^{\mathfrak{x}}(H)$. Note also that every two-generator subgroup of $C_{G}^{\mathfrak{x}}(H)$ is in $\mathfrak{X}$, hence $C_{G}^{\mathfrak{x}}(H)$ also belongs to $\mathfrak{X}$. 
Proposition 2.4. Let $\mathfrak{X}$ be a group theoretical class satisfying $(\star)$. Let $G$

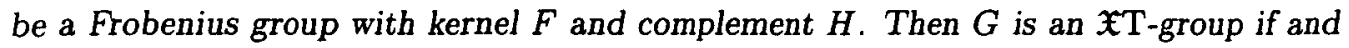
only if $C_{G}^{\mathfrak{x}}(F)$ and $C_{G}^{\mathfrak{x}}(H)$ are $\mathfrak{X}$-groups.

Proof: Let $\mathfrak{X}$ and $G$ be as above. If $G$ is an $\mathfrak{X T}$-group, then it follows from Theorem 2.2 that $F$ and $H$ belong to $\mathfrak{X}$. Consequently $C_{G}^{\mathfrak{x}}(F)$ and $C_{G}^{\mathfrak{x}}(H)$ are also $\mathfrak{X}$ groups by Lemma 2.3. Conversely, suppose that $C_{G}^{\mathfrak{X}}(F)$ and $C_{G}^{\mathfrak{x}}(H)$ are $\mathfrak{X}$-groups. Let $x, y, z \in G \backslash\{1\}$ and suppose that $\langle x, y\rangle \in \mathfrak{X}$ and $\langle y, z\rangle \in \mathfrak{X}$. Assume first that $y \in F$. Then $x, z \in C_{G}^{\mathfrak{x}}(F)$ and consequently $\langle x, z\rangle \in \mathfrak{X}$. If $y \notin F$, then $y \in H^{g}$ for some $g \in G$. But then $x, z \in C_{G}^{\mathfrak{x}}\left(H^{g}\right)=\left(C_{G}^{\mathfrak{x}}(H)\right)^{g}$, thus $\langle x, z\rangle$ belongs to $\mathfrak{X}$. Thus $G$ is an XT-group.

When $\mathfrak{X}$ is the class of all Abelian groups, then all three possibilities of Theorem 2.2 can occur $[\mathbf{6}, \mathbf{1 1}]$. In some cases, however, we can exclude the existence of $\mathfrak{X}$-semisimple

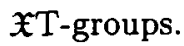

THEOREM 2.5. Let $\mathfrak{X}$ be a class of groups satisfying ( $\star$ ), and suppose that $\mathfrak{X}$ contains all finite dihedral groups and that every finite $\mathfrak{X}$-group is soluble. If $G$ is a finite $\mathfrak{X T}$-group which is not in $\mathfrak{X}$, then $G$ is a Frobenius group with complement belonging to $\mathfrak{X}$. In particular, $G$ is soluble.

Before proving this result we mention here the well known Thompson's classification of minimal simple groups; that is, finite nonabelian simple groups all whose proper subgroups are soluble. It turns out [9] that every such group is isomorphic to one of the following groups.

(i) $\operatorname{PSL}(2, p)$, where $p$ is a prime, $p>3$ and $p^{2}-1 \neq 0 \bmod 5$.

(ii) $\operatorname{PSL}\left(2,2^{f}\right)$, where $f$ is a prime.

(iii) $\operatorname{PSL}\left(2,3^{f}\right)$, where $f$ is an odd prime.

(iv) $\operatorname{PSL}(3,3)$.

(v) $\operatorname{Sz}(q)$, where $q=2^{2 n+1}$ and $2 n+1$ is a prime.

If $G=\operatorname{PSL}(2, F)$ where $F$ is a Galois field of odd characteristic and $|F|>5$, then $G$ can be generated by an involution and an element of even order. This can be easily seen as follows. Let $q=|F|$. By Dickson's theorem [2], $G$ contains elements $a$ and $b$ with $|a|=(q-1) / 2$ and $|b|=(q+1) / 2$. Note that precisely one of $|a|,|b|$ is even, without loss of generality we may assume that this is true for $|a|$. Then $N_{G}(a)=D_{q-1}$ and this is the only maximal subgroup of $G$ containing $a$; this follows from the proof of Dickson's theorem [2]. So if we choose any involution $u$ from $G \backslash N_{G}(a)$, we have $\langle a, u\rangle=G$, as required. A similar result holds true for $\operatorname{PSL}(3,3)$ and $\operatorname{Sz}(q)$. In the first case note that $\operatorname{PSL}(3,3)$ can be generated by the canonical projections of matrices

$$
\left(\begin{array}{ccc}
0 & 0 & 1 \\
0 & -1 & 0 \\
1 & 0 & 0
\end{array}\right) \text { and }\left(\begin{array}{ccc}
0 & 0 & -1 \\
1 & 0 & 0 \\
1 & -1 & 1
\end{array}\right)
$$


which are of orders 2 and 8 in $\operatorname{PSL}(3,3)$, respectively. For the Suzuki groups $\operatorname{Sz}(q)$ it follows from [8] that they can always be generated by an involution and an element of order 4 . We summarise this in the following lemma.

LEMMA 2.6. Let $G$ be one of the following groups: $\operatorname{PSL}(2, F)$ where $F$ is a Galois field of odd characteristic and $|F|>5, \operatorname{PSL}(3,3)$ or $\mathrm{Sz}(q)$. Then $G$ can be generated by an involution and an element of even order.

Note that for the groups $\operatorname{PSL}\left(2,2^{f}\right)$ the conclusion of the above lemma does not hold. In this case we have the following result that can be proved by straightforward calculation.

LEMma 2.7. Let $G=\operatorname{PSL}\left(2,2^{f}\right), f>1$. Denote by $\zeta$ a generator of $\operatorname{GF}\left(2^{f}\right)$ and let $a, b$ and $c$ be the elements of $G$ which are projections of

$$
\left(\begin{array}{ll}
0 & 1 \\
1 & 0
\end{array}\right),\left(\begin{array}{cc}
0 & \zeta \\
\zeta^{-1} & 0
\end{array}\right) \text { and }\left(\begin{array}{cc}
0 & \zeta \\
\zeta^{-1} & \zeta
\end{array}\right)
$$

respectively. Then $\langle a, b\rangle$ and $\langle b, c\rangle$ are dihedral groups and $\langle a, c\rangle=G$.

Proof of Theorem 2.5: We may suppose that $G$ does not belong to $\mathfrak{X}$, hence $R_{\mathfrak{X}}(G) \neq G$. If we prove that $G$ is soluble, then $R_{\mathfrak{X}}(G) \neq 1$ and our claim follows from Theorem 2.2. So suppose that there exist finite insoluble $\mathfrak{X T}$-groups, and let $G$ be a counterexample of minimal order. Then every proper subgroup of $G$ is soluble. By Theorem 2.2 we have that $R_{\mathfrak{X}}(G)=1$. Let $R$ be the soluble radical of $G$. Since $\mathfrak{X}$ contains all finite Abelian groups, we have that $R=1$. It is now easy to see that $G$ has to be simple. By Thompson's classification of minimal simple groups [9], $G$ is isomorphic to one of the groups in the above mentioned list. By Lemma 2.7, $G$ is not isomorphic to any of $\operatorname{PSL}\left(2,2^{f}\right)$, where $f$ is a prime. If $G$ is one of the groups of Lemma 2.6, then $G=\langle a, b\rangle$, where $|a|=2$ and $|b|=2 k, k>1$. We have that $\left\langle a, b^{k}\right\rangle$ is a dihedral group and $\left\langle b^{k}, b\right\rangle$ is a cyclic group, hence $G$ is in $\mathfrak{X}$ by the $\mathfrak{X T}$-property, a contradiction. This concludes the proof.

Using Theorem 2.5, we obtain a rather surprising characterisation of finite soluble groups.

COROLLARY 2.8. Every finite soluble-transitive group is soluble.

Note that the class of all supersoluble groups also satisfies all the assumptions of Theorem 2.5. Thus we have the following.

COROLlary 2.9. Let $G$ be a finite supersoluble-transitive group. If $G$ is not supersoluble, then $G$ is a Frobenius group with supersoluble complement. In particular, $G$ is always soluble.

In view of Corollary 2.8 we may ask if every finite supersoluble-transitive group is supersoluble. This is not true however, as the group $A_{4}$ shows. It is also not difficult 
to find an example of a Frobenius group with supersoluble complement which is not supersoluble-transitive. This example also shows that Proposition 2.4 is in a certain sense best possible. Indeed, it is not possible to replace $C_{G}^{x}(F)$ and $C_{G}^{x}(H)$ by $F$ and $H$, respectively.

Example 2.10. Let $A=\langle x\rangle \oplus\langle y\rangle$ be an elementary group of order 9 and let $\alpha$ be the automorphism of $A$ given by the matrix

$$
\left(\begin{array}{ll}
2 & 2 \\
2 & 1
\end{array}\right) .
$$

Then $\langle\alpha\rangle$ acts fixed-point-freely on $A$. Let $G=A \rtimes\langle\alpha\rangle$. This is a group of order 36 which is not supersoluble-transitive. To see this, note that $\left\langle\alpha^{2},(\alpha y)^{2}\right\rangle$ is a dihedral group, $\left\langle(\alpha y)^{2}, \alpha y\right\rangle$ is cyclic, whereas $\left\langle\alpha^{2}, \alpha y\right\rangle=G$ is not supersoluble. Denoting by $\mathfrak{S}$ the class of all supersoluble groups, note that $C_{G}^{\mathcal{E}}(\langle\alpha\rangle)$ has 20 elements and it is thus not a subgroup of $G$. On the other hand, $C_{G}^{\mathscr{E}}(A)$ is a subgroup of index 2 in $G$.

Theorem 2.5 cannot be applied in the case of $\mathfrak{N T}$-groups, where $\mathfrak{N}$ denotes the class of all nilpotent groups. Thus it is to be expected that there exist finite insoluble $\mathfrak{N T}$ groups. This is confirmed by the following characterisation of finite $\mathfrak{N T}$-groups which is essentially contained in [1]. We include a proof for the sake of completeness.

TheOREM 2.11. Let $G$ be a finite NT-group. Then one of the following holds.

(i) $G$ is nilpotent.

(ii) $G$ is a Frobenius group with nilpotent complement.

(iii) $G \cong \operatorname{PSL}\left(2,2^{f}\right)$ for some $f>1$.

(iv) $G \cong \mathrm{Sz}(q)$ with $q=2^{2 n+1}>2$.

Conversely, every finite group under (i)-(iv) is an NT-group.

Proof: If $G$ is soluble and not nilpotent, then the Fitting subgroup $F$ of $G$ is a proper nontrivial subgroup of $G$. By Theorem $2.2, G$ is a Frobenius group with nilpotent complement. So suppose that $G$ is not soluble. It is easy to see that in every finite NTgroup $G$ the centralisers of nontrivial elements are nilpotent, that is, $G$ is an CN-group. By a result of Suzuki [7, Part I, Theorem 4], the centraliser of any involution in $G$ is a 2-group. Let $P$ and $Q$ be any Sylow $p$-subgroups of $G$ and suppose that $P \cap Q \neq 1$. Since $P$ and $Q$ are nilpotent and $G$ is an NT-group, we conclude that $\langle P, Q\rangle$ is nilpotent. This shows that the Sylow subgroups of $G$ are independent. Combining Theorem 1 in Part I and Theorem 3 in Part II of [7], we conclude that $G$ has to be simple. Additionally, it follows from [8] that $G$ is isomorphic either to $\operatorname{PSL}\left(2,2^{f}\right)$, where $f>1$, or to $\operatorname{Sz}(q)$ with $q=2^{2 n+1}>2$.

Let $G$ be a finite Frobenius group with the kernel $N$ and a complement $H$ and suppose that $H$ nilpotent. Let $x, y, z \in G \backslash\{1\}$ and let the groups $\langle x, y\rangle$ and $\langle y, z\rangle$ be nilpotent. Let $c$ be the nilpotency class of $\langle x, y\rangle$. First suppose that $x \in N$ and $y \notin N$. 
Then $[x, c y]=1$, which implies $\left[x,{ }_{c-1} y\right]=1$, since $H$ acts fixed-point-freely on $N$. By the same argument we get $x=1$, which is not possible. This shows that if $x \in N$ then $y \in N$ and similarly also $z \in N$. But in this case $\langle x, z\rangle$ is clearly nilpotent, since $N$ is nilpotent. Thus we may assume that $x, y, z \notin N$. Let $x \in H^{g}$ and $y \in H^{k}$ for some $g, k \in G$ and suppose $H^{g} \neq H^{k}$. We clearly have $C_{G}(x) \leqslant H^{g}$ and $C_{G}(y) \leqslant H^{k}$. Let $\omega$ be any commutator of weight $c$ with entries in $\{x, y\}$. Then $\omega \in C_{G}(x) \cap C_{G}(y)=1$ implies that $\langle x, y\rangle$ is nilpotent of class $\leqslant c-1$, a contradiction. Hence we conclude that $\langle x, y\rangle \leqslant H^{g}$ and similarly also $\langle y, z\rangle \leqslant H^{g}$. Therefore we have $\langle x, z\rangle \leqslant H^{g}$. But $H^{g}$ is nilpotent, hence the group $\langle x, z\rangle$ is also nilpotent. This shows that the groups under (ii) are $\mathfrak{N T}$-groups.

It remains to prove that the groups under (iii) and (iv) are $\mathfrak{N T}$-groups. If $G$ $=\operatorname{PSL}\left(2,2^{f}\right), f>1$, then every centraliser of a nontrivial element of $G$ is Abelian by [6]. It follows from here that $G$ is an $\mathfrak{N T}$-group. Now let $G=\operatorname{Sz}(q)$ where $q=2^{2 n+1}>2$. By Theorem 3.10 (c) in [3], $G$ has a nontrivial partition $\left(G_{i}\right)_{i \in I}$, where for every $i \in I$ the group $G_{i}$ is nilpotent and contains centralisers of each of its nontrivial elements. Let $x, y, z \in G \backslash\{1\}$ and suppose that the groups $\langle x, y\rangle$ and $\langle y, z\rangle$ are nilpotent. Let $a$ and $b$ be nontrivial elements in $Z(\langle x, y\rangle)$ and $Z(\langle y, z\rangle)$, respectively, and suppose that $a \in G_{i}$ and $b \in G_{j}$ for some $i, j \in I$. Then $y \in C_{G}(a) \cap C_{G}(b) \leqslant G_{i} \cap G_{j}$, hence $i=j$. But now we get $x, z \in G_{i}$ and since $G_{i}$ is nilpotent, the same is true for the group $\langle x, z\rangle$. Hence $G$ is an $\mathfrak{N T}$-group.

\section{REFERENCES}

[1] C. Delizia, P. Moravec, and C. Nicotera, 'Groups in which the bounded nilpotency of two-generator subgroups is a transitive relation', Beiträge Algebra Geom. (to appear).

[2] B. Huppert, Endliche gruppen (Springer-Verlag, Berlin, 1967).

[3] B. Huppert and N. Blackburn, Finite groups III (Springer-Verlag, New York, 1982).

[4] J.C. Lennox, 'Bigenetic properties of finitely generated hyper-(abelian-by-finite) groups', J. Austral. Math. Soc. 16 (1973), 309-315.

[5] D.J.S. Robinson, (Part 1), Finiteness conditions and generalized soluble groups (Springer-Verlag, Berlin, 1972).

[6] M. Suzuki, 'The nonexistence of certain type of simple groups of odd order', Proc. Amer. Math. Soc. 8 (1957), 686-695.

[7] M. Suzuki, 'Finite groups with nilpotent centralizers', Trans. Amer. Math. Soc. 99 (1961), 425-470.

[8] M. Suzuki, 'On a class of doubly transitive groups', Ann. of Math. 75 (1962), 105-145.

[9] J.G. Thompson, 'Non-solvable finite groups all whose local subgroups are solvable', Bull. Amer. Math. Soc. 74 (1968), 383-437.

[10] L. Weisner, 'Groups in which the normaliser of every element except identity is abelian', Bull. Amer. Math. Soc. 31 (1925), 413-416.

[11] Y.F. Wu, 'Groups in which commutativity is a transitive relation', J. Algebra 207 (1998), $165-181$. 
Dipartimento di Matematica e Informatica Università di Salerno

Via Ponte don Melillo

84084 - Fisciano (SA)

Italy

e-mail: cdelizia@unisa.it

Dipartimento di Matematica e Informatica Università di Salerno

Via Ponte don Melillo

84084 - Fisciano (SA)

Italy

e-mail:·cnicotera@unisa.it
Fakulteta za Matematiko in Fiziko

Univerza v Ljubljani

Jadranska 19

1000 Ljubljana

Slovenia

e-mail: primoz.moravec@fmf.uni-lj.si 